\title{
StygoTracing - First experiences with biological tracers in drinking water extraction areas
}

\author{
Susanne van den Berg-Stein ${ }^{\ddagger}$, Diana Burghardt\$, Klaus Schwenkl, Hans Jürgen Hahn‡ \\ ‡ II Department of groundwater ecology, IGÖ GmbH, Koblenz-Landau University, Department of environmental sciences, \\ Koblenz-Landau University, Landau Pfalz, Germany \\ $\S$ Department of groundwater management, Dresden Technical University, Dresden, Germany \\ | Department of environmental sciences, Koblenz-Landau University, Landau Pfalz, Germany
}

Corresponding author: Susanne van den Berg-Stein (vandenberg@groundwaterecology.de)

Received: 06 Oct 2018 | Published: 09 Oct 2018

Citation: van den Berg-Stein S, Burghardt D, Schwenk K, Hahn H (2018) StygoTracing - First experiences with biological tracers in drinking water extraction areas. ARPHA Conference Abstracts 1: e30363. https://doi.org/10.3897/aca.1.e30363

\begin{abstract}
Although groundwater is a highly fragmented habitat, both faunistic and hydraulic interactions exist between different aquifers and between groundwater and surface water.

For the drinking water suppliers, in particular surface water intrusion into groundwater means an increased risk of contamination. Thus risk assessment is essential for quality assurance in drinking water supply. In this context, several questions arise: 1) Where does the water come from?; 2) Are there any hydraulic shortcuts to surface waters?; 3) Are there any hydrological interactions between different aquifers?; 4) What are the catchment borders?
\end{abstract}

Invertebrate fauna may display the strength and the direction of hydrological interactions since the dispersal is linked to hydrological pathways. Our hypothesis is: The more intense the hydrogeological interactions the closer the relationship between individuals and populations.

The degree of relationship can be determined using a kind of paternity test for invertebrates. This is a population genetic, microsatellite-based method called 
StygoTracing. The most important tools of StygoTracing are the primers, which have to be designed for each individual tracer species.

Good tracer species are those, which have a wide distribution and occur with high frequency and are easy to determine. We have chosen three species both stygo- and nonstygobiotic ones. The primers and microsatellite analysis applied proved to be suitable even to distinguish single individuals from each other.

StygoTracing has been successfully tested for water supply systems, but not yet in the field.

Thus seven pilot areas were selected in Bavaria, Baden-Württemberg, Palatinate and Saxony, and sampling was conducted on a total of 186 groundwater, spring and stream sites. Meanwhile, first genetic data are available and initial evaluations reveal plausible correlations on different spatial scales.

\section{Keywords}

StygoTracing, biological tracer, groundwater, hydrological interaction, microsatellites

\section{Presenting author}

Susanne van den Berg-Stein

\section{Presented at}

$24^{\text {th }}$ International Conference on Subterranean Biology 